\title{
Role of spreader flaps in primary rhinoplasty, functional and aesthetic outcomes: a systematic review*
}

\author{
Konstantinos Garefis', Iordanis Konstantinidis', Nikolaos Tsetsos², \\ Maria Garefi ${ }^{3}$, Vasilios Nikolaidis', Konstantinos Markou' \\ Rhinology 60: 2, $92-101,2022$ \\ https://doi.org/10.4193/Rhin21.235
}

1 2nd Academic ORL, Head and Neck Surgery, Department Aristotle University of Thessaloniki, Papageorgiou Hospital, Thessaloniki, *Received for publication: Greece

June 4, 2021

2 Department of ORL, Head and Neck Surgery, G. Papanikolaou Hospital, Thessaloniki, Greece

Accepted: January 26,2022

${ }^{3}$ Department of Pediatric, General Hospital of Veroia, Veroia, Greece

\begin{abstract}
Background: The standard treatment for reconstructing the middle vault of the nose is to use spreader grafts. Recently, an alternative technique using spreader flaps has become widely accepted.
\end{abstract}

Methodology: A literature search was performed in MEDLINE, Science Direct, the Cochrane Library and multiple trial registries. The systematic review included studies evaluating the effectiveness of spreader flaps, with or without comparison to the spreader graft technique, in patients who had undergone primary rhinoplasty.

Results: Thirteen studies with a total of 500 patients met the inclusion criteria and were reviewed systematically. All studies measured breathing function improvement. Additionally, aesthetic improvement/satisfaction was evaluated in seven studies. The use of spreader flaps seems to improve breathing function, as seen in twelve out of thirteen studies. Furthermore, the studies assessing the aesthetic aspect of a primary rhinoplasty showed that spreader flaps can provide satisfactory results. The comparison between spreader flaps and spreader grafts showed similar results in most studies dealing with this topic in both the breathing function improvement and aesthetic improvement/satisfaction domains.

Conclusions: This study is the first systematic review assessing the functional and aesthetic outcomes of spreader flaps in primary rhinoplasty, and it shows encouraging results comparable to those of spreader grafts.

Key words: autospreader, spreader flap, spreader graft, rhinoplasty

\section{Introduction}

Rhinoplasty is an operation aiming to correct a wide range of nasal deformities. These deformities may be of congenital or acquired aetiology (e.g. after an injury or the consequence of a previous nasal operation), causing nasal obstruction and aesthetic problems. In most cases, the reconstruction of the middle vault (MV) is necessary for creating a functional nose and to improve the dorsal aesthetic lines in a primary rhinoplasty ${ }^{(1)}$. Chronic nasal obstruction is among the most frequent symptoms. The causes of chronic nasal obstruction are multiple and complex; however, a significant percentage (13-20\%) is due to nasal valve dysfunction ${ }^{(2-4)}$. The internal nasal valve is defined as the angle located at the junction between the upper lateral cartilage caudal edge and the lower lateral cartilage cephalic edge and their relationships with the septum and the head of the inferior turbinate, forming the narrowest portion of the nasal airway. The angle varies between 10 and 15 degrees $^{(5)}$. Many techniques have been developed for the reconstruction of the internal nasal valve and the MV, as well as for the aesthetic improvement of the dorsal lines. The gold standard method is the spreader graft (SG) technique. Sheen presented this technique for the first time in $1984^{(6)}$. Later, the spreader flap (SF) or autospreader flap technique gained more popularity as an alternative method. Fomon first detailed the technique in 1950 , describing it as a rolling of the upper lateral nasal cartilage towards the septum ${ }^{(7)}$. Wood again described the technique in 1992, while Berkowitz and Oneal presented the term SF in $1998^{(8,9)}$. In the 1990s, the same technique was described in 
different terms, such as Sheyan explaining it as upper lateral cartilage bending ${ }^{(10)}$ and Lerma called it a lapel flap ${ }^{(11)}$. In addition, over time, various authors described modifications of the classic SF technique ${ }^{(12,14-16,19)}$.

The purpose of the present study is to review all the existing evidence related to the effectiveness of the use of SFs in patients undergoing primary rhinoplasty, regarding functional and aesthetic outcomes.

\section{Materials and methods}

\section{Search strategy}

This systematic review was performed according to the PRISMA 2009 checklist. Two reviewers (KG and NT) independently performed a comprehensive literature search in MEDLINE, Science Direct and the Cochrane Library electronic databases from 28 May to 28 June 2020 to identify studies that answered the question of interest. For this purpose, the following free-text terms were used: (spreader flap OR autospreader flap OR autospreader OR upper lateral cartilage flap OR turn-in flap) AND (rhinoplasty OR septorhinoplasty). Additionally, the reviewers searched for eligible trials in multiple trial registries, including clinicaltrial. gov, the European Union (EU) Clinical Trials Register and the International Clinical Trials Registry Platform. The ProQuest Dissertations \& Theses (PQDT) database was searched as a source of grey literature. Extensive hand searches of the references of all the retrieved studies and relevant review articles were also performed. While no year limitation was imposed, only studies published in English were considered for inclusion. As this study was a systemic review of published articles, neither informed consent nor ethics approval was required.

\section{Study selection}

The two reviewers conducted the study selection process independently, and disagreements were resolved by consensus. The study inclusion and exclusion criteria were specified prior to the literature search. The studies that fulfilled the specific criteria were considered suitable for selection if 1) at least one group of patients had been operated on with the SF technique, 2) the study population included exclusively adult patients ( $>18$ years) who underwent primary rhinoplasty, 3) the studies included at least 10patients, 4) the study outcomes included breathing function improvement and/or aesthetic improvement/satisfaction and 5) the outcomes were recorded by an objective and/ or subjective method used in at least two studies. Studies were excluded if patients had a history of 1) a previous rhinoplasty, 2) allergic rhinitis, 3) chronic rhinosinusitis with or without nasal polyps and 4) congenital malformations of the nose.

\section{Data extraction and quality assessment}

Two reviewers (KG and NT) independently reviewed each study according to the predefined criteria for eligibility and extracted data. Any disagreement in the process of study selection or data extraction was resolved through consensus. A predefined form was used to extract data from each study. The following data were recorded from each of the eligible studies: general characteristics (first author, year of publication, country, study design, study sample, sex, age, assessment tool, primary/secondary outcomes, length of follow-up and level of evidence,) and outcome data (primary and secondary outcomes).

The quality of included studies was assessed based on criteria set by the Oxford Centre for Evidence-Based Medicine (OCEBM), and each article was assigned a designated level of evidence ${ }^{(17)}$.

\section{Outcomes}

Breathing function improvement (BFI) was set as a primary study outcome and was assessed using objective methods, such as rhinomanometry, acoustic rhinometry, nasal valve angle measurement in CT scans and/or endoscopic images, and/or by using subjective methods, such as the Nasal Obstruction Symptom Evaluation (NOSE) questionnaire and the Functional Visual Analog Scale (FVAS) score.

The researchers determined aesthetic improvement/satisfaction (AIS) as a secondary outcome, and it was assessed by means of subjective evaluation methods, such as the Aesthetic VAS (AVAS) score, the Rhinoplasty Outcome Evaluation (ROE) questionnaire and a satisfaction questionnaire regarding the aesthetic outcome. Whenever available, statistics such as significance level, standard deviations and width were mentioned. Otherwise, the outcomes were presented in a descriptive way.

\section{Statistical analysis}

The ultimate objective of this research was to culminate in a quantitative synthesis of the main outcome measures. However, the small sample sizes and the lack of reported data combined with the different outcome measures and follow-ups made it impossible to lead to a meta-analysis.

\section{Results}

\section{Literature search}

The systematic search in bibliographic databases yielded 1,455 publications. Four additional records were also identified from trial registries, three of which had already been published and included in the systematic search and one was unpublished, although it was completed. After removing duplicates, 1,352 publications were available for title-abstract screening. Finally, 48 articles that could provide relevant data for answering the research questions were identified. The full texts of these studies were examined thoroughly, resulting in 13 studies that investigated the effectiveness of the SF technique in patients who underwent primary rhinoplasty. The study flow diagram is depicted in Figure 1. 


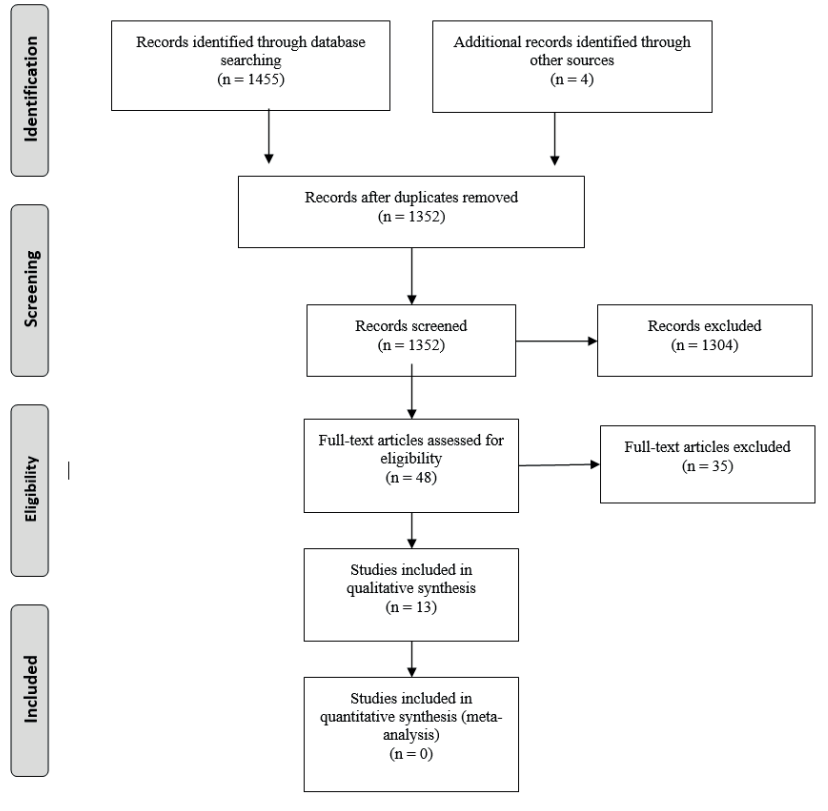

Figure 1. Literature search strategy. PRISMA flowchart detailing literature search and review.

\section{Study characteristics}

Thirteen studies fulfilled the inclusion criteria ${ }^{(12-16,18-25)}$. Of these, three were randomised controlled trials (RCTs), three were clinical trials (randomised or not), five were prospective studies and two were retrospective studies. The studies were published between 2011 and 2020. In total, 500 patients who had undergone primary rhinoplasty were evaluated. In 377 patients, the SF technique exclusively or a SF/SG combination was used. In the remaining 123 patients, who were included in the control groups, the SG technique was used in 89 patients, and another intervention was used in 34 patients. In these 34 patients instead of creation of the spreader flap, the excess upper lateral cartilage was removed. The upper lateral cartilage edge was then repaired and sutured to the septum. The sample sizes of the studies varied between 15 and 66 patients. The characteristics of the 13 included studies, as well as the baseline demographics of the patients, are presented in Table 1.

\section{Breathing function improvement}

The BFI was measured in all 13 included studies s $^{(12-16,18-25)}$ by using objective and subjective methods (Table 2).

\section{Objective methods}

\section{Acoustic rhinometry}

In two studies ${ }^{(12,13)}$, acoustic rhinometry was used as an objective method for the evaluation of BFI. In particular, in the Eren et al. study ${ }^{(12)}$, the minimal cross-sectional area (MCA) and intranasal total volume (ITV) were increased postoperatively; however, they did not mention the significance level of $\mathrm{p}$. In the Saedi et al. study ${ }^{(13)}$, the MCA differences in both the control and SF groups showed an increase in the left side and a decrease in the right side. However, their differences were not statistically significant $(p=0.50)$.

\section{Rhinomanometry}

In four studies ${ }^{(19,20,24,25)}$, rhinomanometry was used. Görgülü et al. ${ }^{(19)}$ showed a significant decrease in both inhalation and exhalation nasal airway resistance (NAR; $p<0.001)$ by using an active anterior rhinomanometry. In the Hassanpour et al. study ${ }^{(20)}$, NAR increased significantly and flow rate (FR) decreased significantly postoperatively in both groups (SF and SG; all $p \leq 0.05$ ). However, no statistically significant difference was observed between the two groups (NAR difference $p=0.19$ and FR difference $p=0.07$, respectively). Rezaei et al. ${ }^{(24)}$ showed that the average FR and NAR in inhalation and exhalation had no significant difference postoperatively between the SF and SG groups. Inhalation FR on the left side and exhalation NAR on the right side were significantly reduced postoperatively in the SG group ( $p=0.025$ and $p=0.04$, respectively). Finally, in the Zeid et al. study ${ }^{(25)}$, a statistically significant reduction in NAR, on the right and left sides, was observed six months postoperatively in both groups (SF and SG; $\mathrm{p}<0.001$ ), with no significant difference between them.

\section{CT scan images}

Hussein et al. measured the nasal valve angle on CT scan images $^{(14)}$. An increase in the postoperative angle was shown in both groups (nasal obstruction group and without symptoms group). However, the level of statistical significance was not mentioned.

\section{Endoscopic images}

In the Hussein et al..$^{(14)}$ and Kocak et al. ${ }^{(22)}$ studies, the angle of the nasal valve was evaluated pre-or intra- and postoperatively by endoscopic images. Adobe Photoshop was used to calculate the angles. Hussein et al. ${ }^{(14)}$ presented an increase of the angle postoperatively without mentioning the level of statistical significance. Kocak et al. ${ }^{(22)}$ presented a statistically significant increase in the nasal valve angle postoperatively in all three study groups $(p=0.00)$. Although, the highest postoperative improvement was observed in the SF group with triangular SG $(p=0.001)$.

\section{Subjective methods}

\section{NOSE score}

The NOSE questionnaire was used as one of the basic subjective methods for evaluating BFI in eight ${ }^{(12,14,15,16,18,21-23)}$ of the 13 studies ${ }^{(12-16,18-25)}$. Specifically, all studies noted an improvement in breathing function. However, this improvement was statistically significant in six ${ }^{(12,15,18,21-23)}$ of the eight studies ${ }^{(12,14,15,16,18,21-23)}$. Particularly, Yoo et al. ${ }^{(18)}$ presented a significant BFI in the group with nasal obstruction $(p<0.001)$. In the Kocak et al. study ${ }^{(22)}$, a significant reduction in the NOSE score was observed in the SF group and in the SF group with triangular SG $(p=0.05)$. There was no significant postoperative difference between the study's groups ( $p=0.695)$. Similarly, in the Sowder et al. study ${ }^{(21)}$, no dif- 


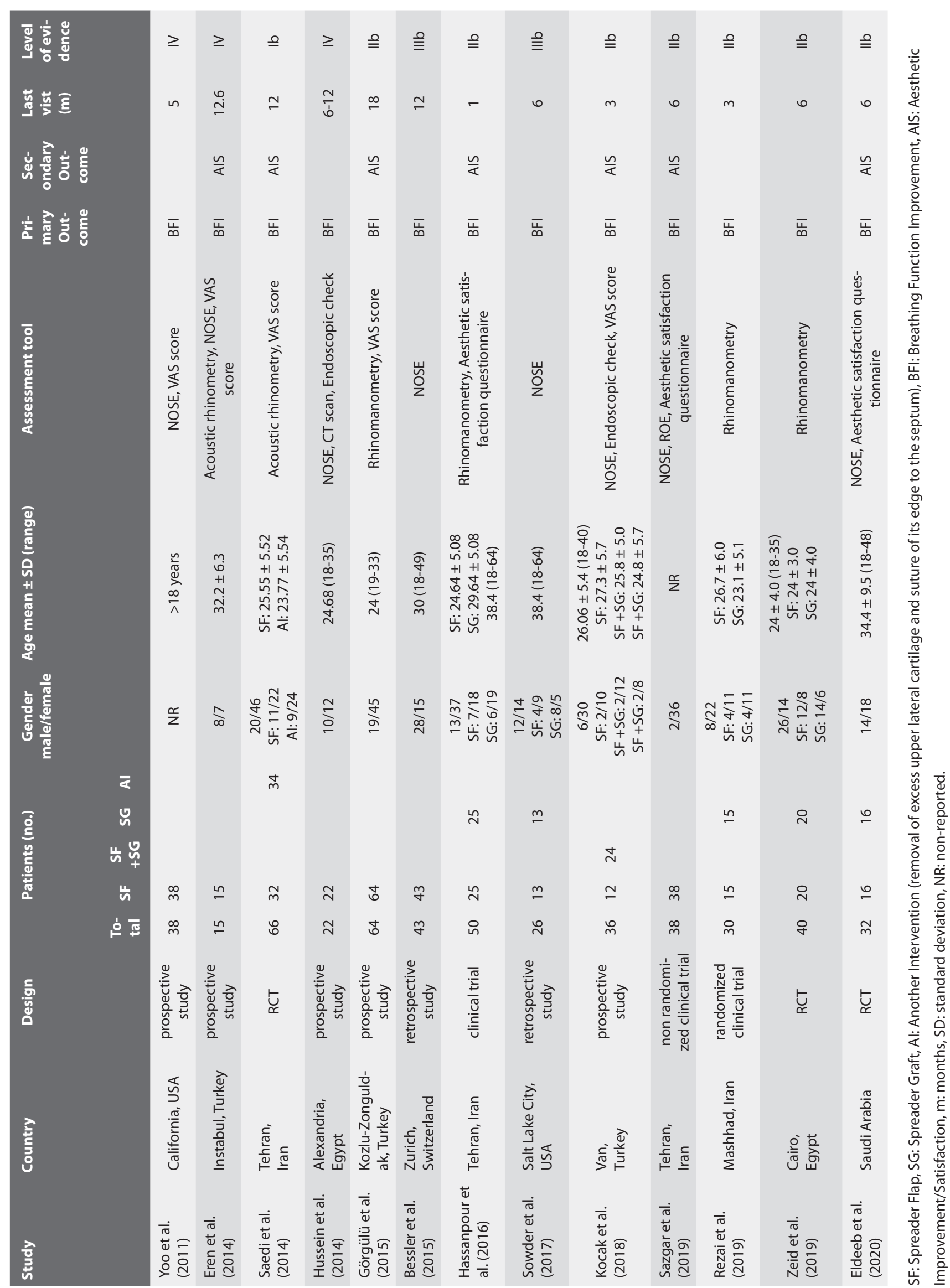




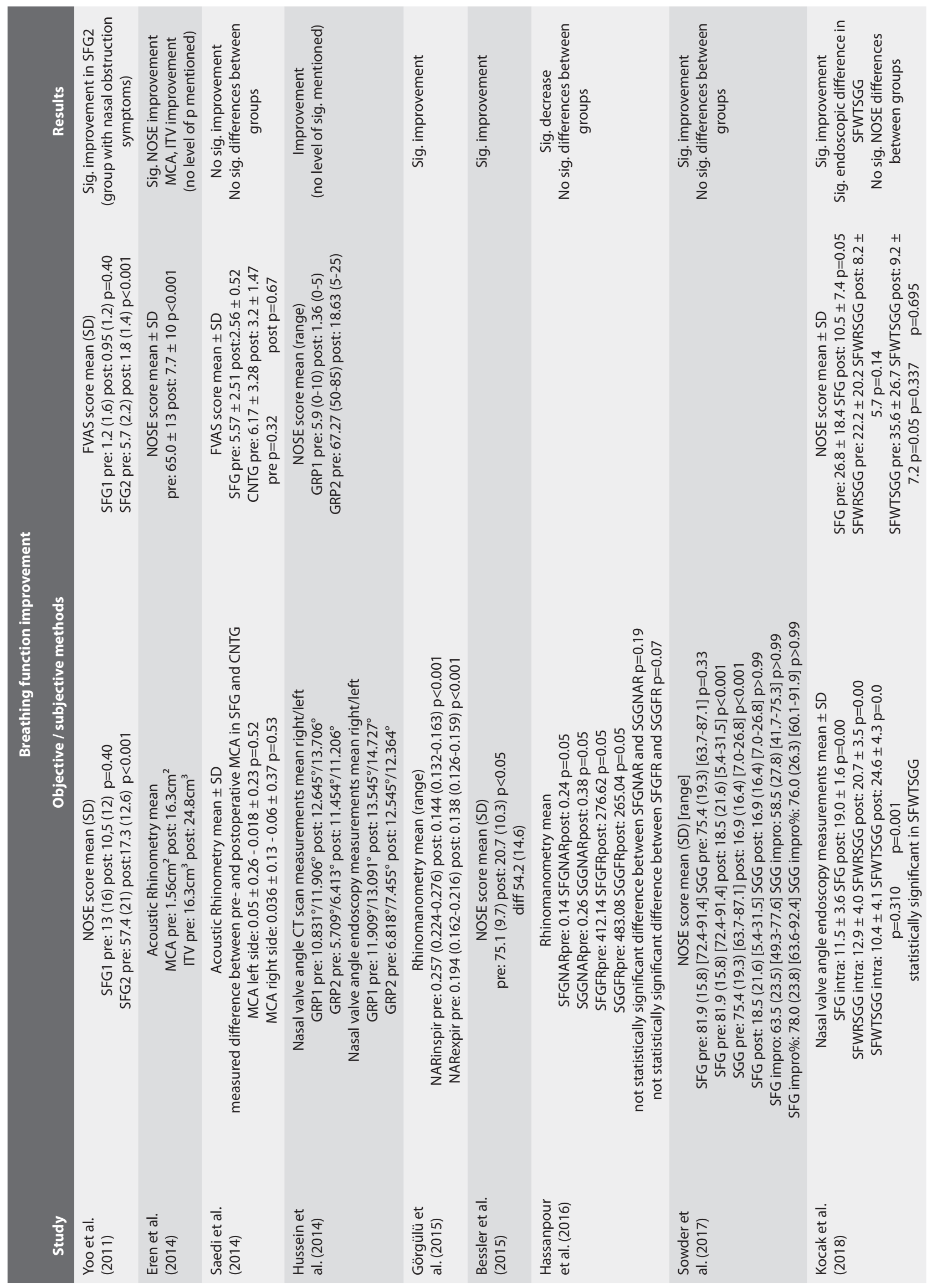




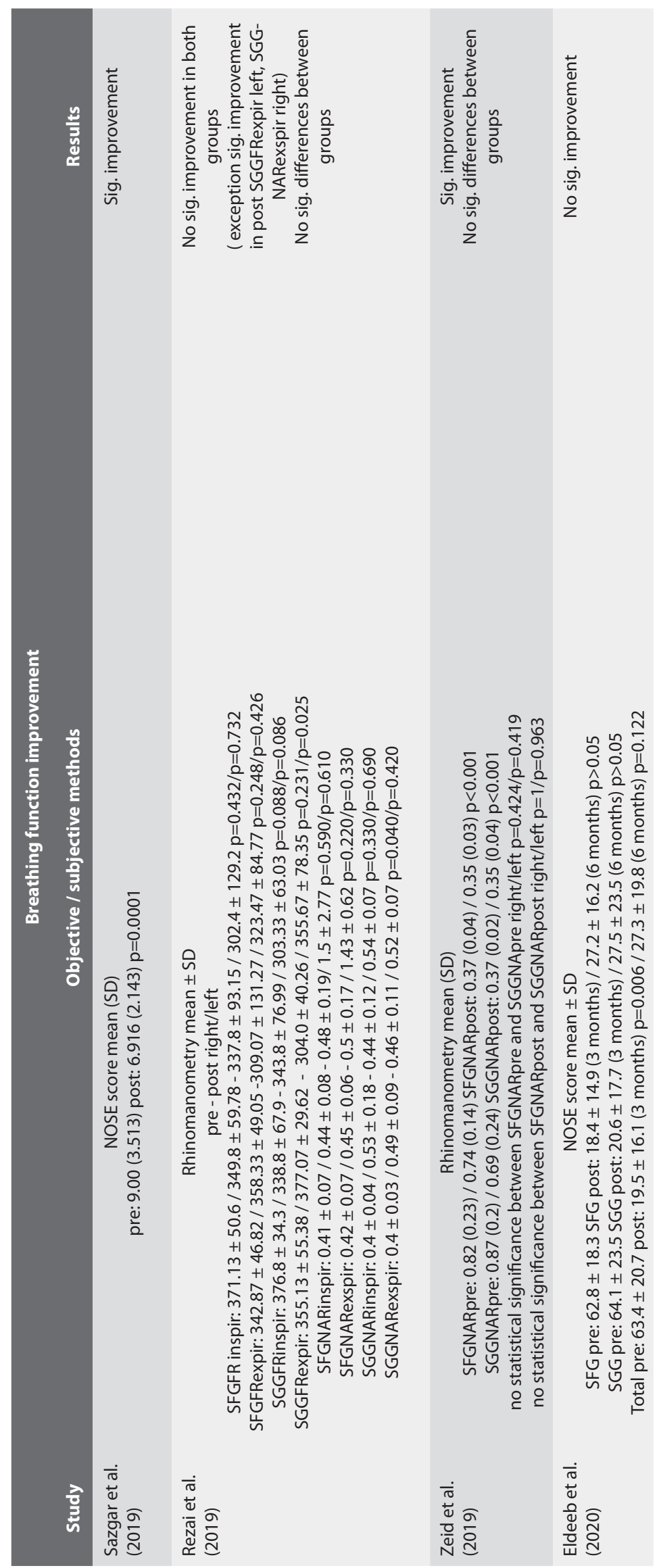

SFG: spreader flap group, SGG: spreader graft group, SFG1: group without nasal obstruction symptoms, SFG2: group with nasal obstruction symptoms, CNTG: control group received another intervention, MCA $\left(\mathrm{cm}^{2}\right)$ : minimal cross-sectional area, ITV $\left(\mathrm{cm}^{3}\right)$ : total intranasal volume, GRP1: group without nasal obstruction symptoms, GRP2: group with nasal obstruction symptoms, NAR $\left(\mathrm{Pa} / \mathrm{cm}^{3} / \mathrm{s}\right)$ : nasal airway resistance, inspir : inspiratory, expir: expiratory, FR: flow rate, SFWRSGG: spreader flap with rectangular spreader graft group, SFWTSGG: spreader flap with rectangular spreader graft group, pre: preoperative, post: postoperative, intra: intraoperative, impro: improvement, SD: standard deviation.

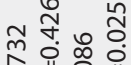

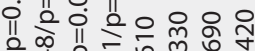

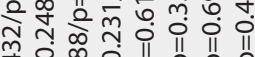
(11)

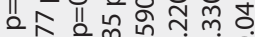

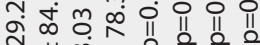

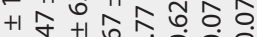

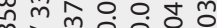
is

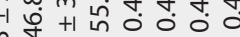

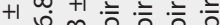
t. $\because$.

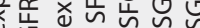
突 
Table 3. Summary of studies' aesthetic results

\section{Aesthetic improvement/satisfaction}

(2011)

Sig. improvement

$\begin{array}{lc}\text { Eren et al. } & \text { AVAS score mean } \pm \text { SD } \\ \text { (2014) } & \text { pre: } 32.0 \pm 20 \text { post: } 88 \pm 9 p<0.001 \\ \text { Saedi et al. } & \text { AVAS score mean } \pm \text { SD } \\ \text { (2014) } & \text { SFG pre: } 4.43 \pm 2.49 \text { post: } 8.38 \pm 1.6 \\ & \text { CNTG pre: } 3.83 \pm 1.72 \text { post: } 8.68 \pm 0.95 \\ & \text { pre } p=0.005 \quad \text { post } p=0.38\end{array}$

(2014)

(2014)

\begin{tabular}{|c|c|c|c|c|c|c|}
\hline \multicolumn{7}{|l|}{$\begin{array}{l}\text { Hussein et } \\
\text { al. (2014) }\end{array}$} \\
\hline \multicolumn{7}{|l|}{$\begin{array}{l}\text { Görgülü et } \\
\text { al. (2015) }\end{array}$} \\
\hline \multicolumn{7}{|l|}{$\begin{array}{l}\text { Bessler et } \\
\text { al. (2015) }\end{array}$} \\
\hline \multicolumn{7}{|l|}{$\begin{array}{l}\text { Hassan- } \\
\text { pour et al. } \\
\text { (2016) }\end{array}$} \\
\hline \multicolumn{7}{|l|}{$\begin{array}{l}\text { Sowder et } \\
\text { al. (2017) }\end{array}$} \\
\hline $\begin{array}{l}\text { Kocak et al. } \\
\text { (2018) }\end{array}$ & \multicolumn{6}{|c|}{$\begin{array}{c}\text { AVAS score mean } \pm \text { SD } \\
\text { SFG pre: } 4.00 \pm 1.9 \text { SFG post: } 8.8 \pm 0.7 p \\
\text { SFWRSGG pre: } 4.07 \pm 1.8 \text { SFWRSGG post: } 8.8 \pm \\
\text { SFWTSGG pre: } 3.3 \pm 1.5 \text { SFWTSGG post: } 8.7 \pm \\
p=0.536 \quad p=0.910\end{array}$} \\
\hline \multirow[t]{6}{*}{$\begin{array}{l}\text { Sazgar et } \\
\text { al. (2019) }\end{array}$} & \multicolumn{6}{|c|}{$\begin{array}{l}\text { ROE } \\
\text { Question No Slightly Moderately } \\
\text { Very Extremely }\end{array}$} \\
\hline & 1 & 1 & 3 & 13 & 6 & 13 \\
\hline & & 2 & 3 & 9 & 7 & 15 \\
\hline & & 0 & 7 & 8 & 7 & 14 \\
\hline & 4 & 8 & 7 & 7 & 8 & 6 \\
\hline & 5 & 1 & 2 & 7 & 9 & \\
\hline
\end{tabular}

Rezai et al.

(2019)

Zeid et al. (2019)

Eldeeb et al. (2020)
NR

NR

NR

\begin{tabular}{lcc}
\multicolumn{3}{c}{ Satisfaction questionnaire } \\
& SFG & SGG \\
complete satisfied: & 10 & 8 \\
partially satisfied: & 11 & 14 \\
unsatisfied: & 4 & 3
\end{tabular}

SFG: $60 \%$ complete or partially satisfied

SGG: $62,9 \%$ complete or partially satisfied

NR

NR

Sig. improvement No sig. differences between groups $\begin{array}{cc}\text { Satisfaction questionnaire } & 97,1 \% \text { complete or } \\ \text { complete satisfied:19 } & \text { partially satisfied }\end{array}$ partially satisfied:16 unsatisfied:1
NR

NR
NR

NR

SFG: $68,8 \%$ complete or partially satisfied SGG: 93,8\% complete or partially satisfied Sig. difference in SGG

statistically significant difference between SFG/SGG p $=0.038$

SFG: spreader flap group, SGG: spreader graft group, CNTG: control group received another intervention, SFWRSGG: spreader flap with rectangular spreader graft group, SFWTSGG: spreader flap with rectangular spreader graft group, pre: preoperative, post: postoperative, NR: non-reported. SD: Standard deviation.

ference was found in postoperative NOSE scores between the SF and SG groups ( $p>0.99)$. Hussein et al. ${ }^{(14)}$ showed a postoperative improvement in the subjective nasal airway patency but failed to mention the significant level of $p$ value. Lastly, in the Eldeeb et al. study ${ }^{(16)}$, there was a great improvement in the patients' symptoms of nasal obstruction, which was statistically insignificant $(p>0.05)$.

\section{Functional VAS score}

In two studies ${ }^{(13,18)}$, BFI was evaluated by means of the FVAS score. Specifically, in the You et al. study ${ }^{(18)}$, the VAS score was re- 
duced in both SF groups. However, the SF group with nasal obstruction symptoms showed a statistically significant postoperative decrease $(p<0.001)$. Saedi et al. ${ }^{(13)}$ observed a postoperative $\mathrm{BFI}$ in both the SF and control groups; however, the significance level was not mentioned. Finally, the differences between preoperative and postoperative FVAS scores in both groups were not statistically significant ( $p=0.32$ and $p=0.67$, respectively).

Aesthetic improvement/satisfaction

Seven included studies used subjective measures to assess AIS

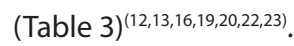

\section{Subjective methods}

\section{Aesthetic VAS score}

The rhinoplasty's aesthetic results were evaluated with an AVAS score in four studies ${ }^{(12,13,19,22)}$. In two of the studies ${ }^{(12,19)}$, a significant postoperative increase of the VAS score $(p<0.001)$ was noted. In the Saedi et al. study ${ }^{(13)}$, patients in both the SF and control groups were satisfied with the aesthetic results. However, the significance level was not reported. Furthermore, the preoperative difference in the AVAS score between the groups was statistically significant ( $p=0.005)$. On the contrary, the postoperative difference was not significant $(p=0.38)$. Finally, in all three groups of the Kocak et al. study ${ }^{(22)}$, the AVAS score postoperatively increased significantly $(p=0.00)$, with no statistically significant difference between them $(p=0.910)$.

ROE-Satisfaction questionnaires

In three studies ${ }^{(16,20,23)}$, patient satisfaction regarding the aesthetic outcome of the rhinoplasty was assessed through a satisfaction questionnaire, and in only one was the ROE questionnaire used $^{(23)}$. In particular, in the Hassanpour et al. study ${ }^{(20)}$, the vast majority of patients in both intervention groups were satisfied or moderately satisfied with the aesthetic outcome. Eldeeb et al. ${ }^{(16)}$ showed a statistically significant higher satisfaction $(p=0.038)$ in patients who had received the SG intervention in comparison to those who had received the SF intervention. Lastly, in the Sazgar et al. study ${ }^{(23)}$, patients were postoperatively satisfied with the aesthetic result, as recorded in both the rhinoplasty satisfaction and ROE questionnaires.

\section{Level of evidence}

Three $(12,14,18)$ of the included studies were graded as level IV evidence, and two ${ }^{(15,21)}$ studies were graded as level IIlb. The majority ${ }^{(16,19,20,22-25)}$ of this systematic review's studies (seven of 13) were graded as level Ilb evidence, and only one study ${ }^{(13)}$ was graded as level Ib (Table 1).

\section{Discussion}

The present study is the first systematic review assessing the effectiveness of SFs, in their classic or modified form, in patients undergoing primary rhinoplasty in comparison to SGs. This systematic review resulted in two major conclusions: 1. The use of SFs seems to improve nasal breathing function, as seen in twelve out of thirteen studies ${ }^{(12-16,18,19,21-25)}$, by reconstructing the internal nasal valve and the MV.

2. The SFs can provide satisfactory results regarding the aesthetic outcome of the primary rhinoplasty, as seen in seven stu$\operatorname{dies}^{(12,13,16,19,20,22,23)}$, through the restoration of the dorsal aesthetic lines.

BFI after the use of SFs was statistically significant in eight studies $^{(12,15,18,19,21-23,25)}$. The remaining four studies ${ }^{(13,14,16,24)}$ reported

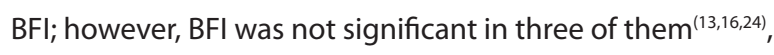
and one did not mention the significance level ${ }^{(14)}$. The only exception was the Hassanpour et al. ${ }^{(20)}$ study where the authors found a significant decrease of nasal breathing function in both techniques. It was attributed to the short follow up period. In addition, six studies ${ }^{(16,20-22,24,25)}$ compared the effectiveness of SFs vs SGs regarding functional outcome. According to four ${ }^{(20,21,24,25)}$ out of these six studies ${ }^{(16,20-22,24,25)}$, there was no significant difference in improvement between the two techniques. In the Kocak et al. study ${ }^{(22)}$, the combined triangular SG with SF was the better technique for widening the nasal valve angle than SF alone. Furthermore, Eldeeb et al. ${ }^{(16)}$ did not report BFI differences between the two techniques; however, the significance level was not mentioned.

A significant aesthetic improvement with the SF technique was shown in three ${ }^{(12,19,22)}$ of the seven studies ${ }^{(12,13,16,19,20,22,23)}$. In one study ${ }^{(13)}$, there was aesthetic improvement, although the significance level was not mentioned. In the remaining three studies ${ }^{(16,20,23)}$, the vast majority of patients were complete or partially satisfied with the aesthetic outcome. Regarding AIS, three studies ${ }^{(16,20,22)}$ compared SF and SG techniques. No significant differences in AIS were reported between the two techniques in one ${ }^{(22)}$ of the three studies $(16,20,22)$. Additionally, Hassanpour et al. ${ }^{(20)}$ reported similar satisfactory results but without mentioning the significance level. Only one study(16) showed that the SG technique had a better aesthetic outcome compared with the aesthetic outcome of the SFs.

Rhinoplasty is not only an aesthetic operation but also a functional one, as the MV and the nasal valve have a significant role in nasal airflow. SFs are a simple and fast technique that uses the existing structures; therefore, there is no need for additional grafts. Besides SFs and SGs, there are also additional techniques, such as batten grafts, butterfly grafts and special suture techniques, used to manage nasal valve collapse. The goal of these techniques is to widen the existing angle of the nasal valve, enhancing the underlying framework and protecting it from collapse during inhalation ${ }^{(5)}$.

The positive results of the SFs, in both functional and aesthetic outcomes, and the similar SF and SG values are also in accordance with a series of studies that did not satisfy our inclusion criteria $^{(26-28)}$. Zeina et al. ${ }^{(29)}$ assessed patients undergoing septorhinoplasty and reported that both SF and SG techniques resulted in significant radiological and clinical improvement of nasal 
valve obstruction. In addition, Barone et al. ${ }^{(30)}$ showed that there was higher long-term benefit in patients where both techniques were used simultaneously, compared with those operated on with one sole technique. These results are in accordance with the study by Kocak et al. ${ }^{(22)}$ included in the present review. After assessing the evidence levels of included studies (individual evidence grading is available for review), we found that most of them (eight out of 13) had a level higher than IIlb. The relatively high level of evidence strengthens the conclusion of the present systematic review. However, the results of our study are liable to certain limitations. Specifically, there were only a few RCTs with few patients and high heterogeneity. Thus, studies with a wide range of designs that fulfilled the inclusion criteria were included. Research in rhinoplasty is more vulnerable to selection and allocation bias of patients. Furthermore, the studies' limitations are the short period of postoperative follow-up (especially in Hassanpour et al. study ${ }^{(20)}$ ), the differences between studies regarding the SF surgical technique and the type of surgical intervention. More specifically, some authors ${ }^{(15,16)}$ used anterior SFs, where the caudal end of the upper lateral cartilage is folded inwardly. In other studies ${ }^{(12,14,19)}$, the suturing techniques of the SFs differed (modified SF, autospreading spring flap), while in the Kocak et al. study ${ }^{(22)}$, there was a combination of SFs and SGs. Regarding the type of surgical interventions, there was no absolute homogeneity, as in some studies the rhinoplasty had been performed with or without removal of the nasal hump or/and septoplasty or/and reduction of the inferior turbinate. Therefore, it is impossible to determine the contribution of each intervention separately to the final BFI. Additionally, the objective measurements used (rhinomanometry, acoustic rhinometry, CT scan) provide limited information regarding the collapsibility of the nasal valve. Future studies using four-phase rhinomanometry are needed to confirm the value of SFs/SGs after nasal valve reconstruction. Lastly, all the questionaries (NOSE, VAS, ROE) used were validated and widely used, except the satisfactory questionary.

Most of the studies in the systematic review examined the effectiveness of the SFs separately or in comparison to that of the
SGs. However, there is no study comparing the effectiveness of the classic SF technique with modified forms (anterior SF and modified SF). Furthermore, RCT studies are needed with homogeneous groups of patients using a combination of objective (four-phase rhinomanometry and acoustic rhinometry) and subjective methods (NOSE and ROE questionnaires) to assess functional and aesthetic outcomes.

\section{Conclusion}

The use of SFs is a simple, safe, and effective alternative technique for the reconstruction of the MV, nasal valve and dorsal aesthetic lines of the nose in patients undergoing primary rhinoplasty. Despite the limited studies, their heterogeneity, their lack of high-quality RCTs and their different methodological limitations, the functional and aesthetic outcomes of SFs are considered particularly encouraging, not inferior to those of SGs. However, further RCTs evaluating the effectiveness of the SF technique are needed to establish its value in primary rhinoplasty.

\section{Acknowledgements}

This study was not supported by any company or persons other than the authors.

\section{Authorship contribution}

KG: Literature search, data extraction, analysis and manuscript production, IK: Design, analysis and manuscript review, NT:

Design, analysis and manuscript review, MG: Data extraction and analysis, VN: Manuscript review and preparation, KM: Design and expert manuscript review.

\section{Conflict of interest}

All authors declare that there are no conflicts of interest to be reported.

\section{Funding}

Researchers did not receive any specific grant from funding agencies in the public, commercial, or not-for-profit sectors.

\section{References}

1. Kovacevic M, Wurm J. Spreader flaps for middle vault contour and stabilization. Facial Plast Surg Clin N Am. 2015;23; 1-9.

2. Helal MZ, El-Tarabishi M, Sabry SM, et al. Effects of rhinoplasty on the internal nasal valve: a comparison between internal continuous and external perforating osteotomy. Ann Plast Surg. 2010;64(5); 649-57.

3. Schlosser RJ, Park SS. Functional rhinoplasty Operative techniques in otolaryngology. Head Neck Surg. 1999;10(3); 203-8.

4. Elwany $\mathrm{S}$, Thabet $\mathrm{H}$. Obstruction of the nasal valve. J Laryngol Otol. 1996;110(3); 221-4.

5. Friedman 0 . The nasal valves. In Nasal
Physiology and Pathophysiology of Nasal Disorders. Berlin, Heidelberg: Springer, 2013: 281-91.

6. Sheen JH. Spreader graft: a method of reconstructing the roof of the middle nasal vault following rhinoplasty. Plast Reconstr Surg. 1984;73(2); 230-9.

7. Fomon S, Gilbert JG, Caron AL, et al. Collapsed ala: pathologic physiology and management. Arch Otolaryngol. 1950;51; 465-84.

8. Wood WG. Using the upper lateral cartilage as a spreader graft. In Poster presented at the American Society of Plastic Surgeons meeting 1992 Dec.
9. Oneal RM, Berkowitz RL. Upper lateral cartilage spreader flaps in rhinoplasty. Aesthet Surg J. 1998;18(5); 370-1.

10. Seyhan A. Method for middle vault reconstruction in primary rhinoplasty: upper lateral cartilage bending. Plast Reconstr Surg. 1997;100(7); 1941-3.

11. Lerma J. The "lapel" technique. Plast Reconstr Surg. 1998;102(6); 2274.

12. Eren SB, Tugrul S, Ozucer B, et al. Autospreading spring flap technique for reconstruction of the middle vault. Aesthetic Plast Surg. 2014;38(2); 322-8.

13. Saedi B, Amaly A, Gharavis $V$, et al. Spreader flaps do not change early functional out- 
comes in reduction rhinoplasty: a randomized control trial. Am J Rhinol Allergy. 2014;28(1); 70-4.

14. Hussein WK, Elwany S, Montaser M Modified autospreader flap for nasal valve support: utilizing the spring effect of the upper lateral cartilage. Eur Arch OtoRhino-L. 2015;272(2); 497-504.

15. Bessler S, Haemmig HK, Schuknecht B, et al. Anterior spreader flap technique: A new minimally invasive method for stabilising and widening the nasal valve. J Plast Reconstr Aesthet Surg. 2015;68(12); 1687 93.

16. Eldeeb $W$, Naguib MB, Rifaat $M$, et al. A Comparative Study of Open Septorhinoplasty with Spreader Graft and Auto-Spreader Flap in Patients with Nasal Obstruction. ASOL. 2020;2(6); 20-8.

17. OCEBM Levels of Evidence Working Group. (2011) The Oxford Levels of Evidence. URL http://www.cebm.net/ [accessed on June 2020].

18. Yoo S, Most SP. Nasal airway preservation using the autospreader technique: analysis of outcomes using a disease-specific quality-of-life instrument. Arch Facial Plast Surg. 2011;13(4); 231-3.

19. Görgülü, T, Özer CM, Kargi E. The accordion suture technique: a modified rhinoplas ty spreader flap. J Craniomaxillofac Surg. 2015;43(6); 796-802.

20. Hassanpour SE, Heidari A, Moosavizadeh SM, et al. Comparison of aesthetic and func tional outcomes of spreader graft and autospreader flap in rhinoplasty. World J Plast Surg. 2016:5(2); 133-8.
21. Sowder JC, Thomas AJ, Gonzalez CD, et al. Use of spreader flaps without dorsal hump reduction and the effect on nasal function. JAMA Facial Plast Surg. 2017;19; 287-92

22. Kocak OF, Düzenli U. Assessment of Vertical Triangular Spreader Graft Technique for Reconstruction of Middle Vault and Internal Nasal Valve Angle. Arch Craniofac Surg. 2018;29(8); 2096-100.

23. Sazgar AA, Razmara N, Razfar A, et al. Outcome of rhinoplasty in patients undergoing autospreader flaps without notable dorsal hump reduction: A clinical trial. Plast Reconstr Aesthet Surg. 2019;72(10); 1688-93.

24. Rezaei E, Beiraghi-Toosi A, Parand A, et al A randomized clinical trial on efficacy of respiration after rhinoplasty: Comparison between spreader grafts and cartilage flaps. Turk J Plast Surg. 2019;27(4); 199-203.

25. Zeid NG, Mohamed AS, ElSayed ElFouly $M$, et al. Objective Comparison Between Spreader Grafts and Flaps for Mid-Nasal Vault Reconstruction: A Randomized Controlled Trial. Plast Surg (Oakv). 2020 Aug;28(3):137-141.

26. Manavbaşı YI, Başaran I. The role of upper lateral cartilage in dorsal reconstruction after hump excision: Section 1. Spreader flap modification with asymmetric mattress suture and extension of the spreading effect by cartilage graft. Aesthetic Plast Surg 2011;35; 487-93.

27. Mustafa SO. The Role of Autospreader Flap in Primary Rhinoplasty. Iraqi Postgraduate Medical Journal. 2015;14(2); 185-91.

28. Shafaeei Y, Zare NJ. A Comparison of the
Aesthetics Outcomes and Respiratory Side Effects of the Use of Spreader Flap and Spreader Graft Techniques in Open Rhinoplasty. J Craniofac Surg. 2019;30(8); 2546-8.

29. Zeina AM, El Zeheiry AM, El-Din AMB. True and Average Internal Nasal Valve Area in Septorhinoplasty: Radiological and Clinical Outcomes. Ann Plast Surg. 2020;84(5); $487-$ 93.

30. Barone M, Cogliandro A, Salzillo R, et al. Role of Spreader Flaps in Rhinoplasty: Analysis of Patients Undergoing Correction for Severe Septal Deviation with Long-Term FollowUp. Aesthetic Plast Surg. 2019;43(4):10061013

\section{Konstantinos Garefis MD, MSc}

2nd Academic ORL -

Head and Neck Surgery Department

Aristotle University of Thessaloniki

Papageorgiou Hospital

Ring Road, N. Efkarpia

Thessaloniki

Greece

Tel.: +0030 2333024812, +30

6978436964

Fax: +30 23333024037

E-mail:kgarefis@hotmail.com 